\title{
THE AFTER HISTORY OF CASES OF PLEURISY WITH SEROUS EFFUSION IN CHILDREN
}

BY

\author{
D. W. SMITHERS, M.B.; B.CHIR.,
}

Late Clinical Assistant to the Children's Department, St. Thomas's Hospital.

Incidence of the disease.-Serous pleural effusions are most commonly seen in patients in early adult life. In children they occur more commonly towards the latter part of childhood. They are not common in very young children, being rarely seen before the age of three, at which time empyema is comparatively common. Scheel and Föien ${ }^{1}$, in a review of 957 cases of pleurisy, found the largest number between the ages of 16 and 25 . $\mathbf{M a z z e o}^{2}$, examining the incidence of pleural effusions in children up to the age of 12, found the highest percentage to occur between the ages of 6 and 8 years.

Between 1920 and 1932 inclusive 320 cases diagnosed as pleural effusion were admitted to St. Thomas's Hospital : 71 (22 per cent.) were in-patients under 15 years of age, and 5 (1.6 per cent.) in-patients under 5 years of age. There were only two cases in children under 3 (there being 31 cases of empyema in children under 3 over the same period).

Prognosis and sequelae.-The prognosis, both immediate and remote, is much better in children than in adults owing to a greater proportion of the effusions in adults being secondary to gross parenchymatous lesions of the lung.

The immediate prognosis in children is good. There was no fatality while in hospital among the 71 cases under the age of 15 admitted to St. Thomas's Hospital between 1920 and 1932.

The more remote prognosis is not so satisfactory. In adults between 30 and 50 per cent. subsequently develop tuberculosis. Osler ${ }^{3}$ quotes several series of cases followed by Bowditch, Hedges, Cabot, and Hamman in which between 30 and 50 per cent. subsequently became tuberculous, and many other investigators have reported similar results. Hebert ${ }^{4}$ states that about 40 per cent. of pleurisies with effusion are followed by definite pulmonary tuberculosis. Approaching the subject from another angle, Norris and Landis ${ }^{5}$ quote the Records of the Phipps Institute which show that of 5,895 patients in whom a diagnosis of tuberculosis was made, 23.8 per cent. gave an antecedent history of pleurisy. Of the late results in 
children there is less evidence in the literature. Graham ${ }^{6}$ published a series in 1925 and was able to trace only two other such contributions, one by Nobel and Steinebach ${ }^{7}$ in 1921 and the other by Neuland ${ }^{8}$ in 1922. Nobel and Steinebach followed 39 cases, 10 per cent. of which at most subsequently became tuberculous. Neuland followed 24 cases, 16.7 per cent. of which subsequently developed tuberculous lesions. Graham followed 39 cases, 17.9 per cent. of which became tuberculous. The deaths from tuberculosis in these series were 2.5 per cent., none, and 5.1 per cent. respectively. In those children (number not stated) whom Mazzeo $^{2}$ was able to follow over a period of from seven to eight years, no form of pulmonary tuberculosis ever occurred, and other specific tuberculous manifestations only occurred in 2 to 3 per cent. of cases. This led him to regard serous pleurisy in the light of a benignant " episode," with regard to future tuberculous developments, which acted by increasing the specific immunity of the patient. He adds, however, that tuberculous pleurisy is the warning of the presence of an infection which had been latent and might become dangerous if the application of those measures upon which the therapy of tuberculosis relies were neglected. His results are not in accordance with those of this investigation and though raised immunity may play some part in lowering the subsequent tuberculosis rate, it is probably due as much to the recognition of the disease by the appearance of an effusion and subsequent efficient supervision and treatment that subsequent tuberculosis is not more common than it is.

Those cases that do subsequently develop tuberculosis tend to do so within the first few years following the pleurisy. Scheel and Föien ${ }^{1}$ in a report on 957 cases of simple pleurisy, mostly with effusion, found that the mortality from tuberculosis was greatest during the first four years following the pleurisy. Hebert ${ }^{4}$ says that pulmonary tuberculosis develops especially during the five years following pleural effusion. The same holds good in children. In Graham's series, two died of generalized tuberculosis, both one year after the pleurisy; one developed spinal caries in one year, one spinal caries in eight months, one tuberculous empyema in six months, and one tuberculous peritonitis in seven years.

Findlay ${ }^{9}$ reported a case of intrathoracic tuberculous abscess occurring in a boy aged 6 eighteen months after a serous pleural effusion. In Neuland's series three showed definite signs of pulmonary tuberculosis and one of spinal caries. Pulmonary tuberculosis appears to be a less common sequel in children than in adults, as would be expected from the fact that so many more adult cases of pleural effusion are due to direct extension from a pre-existing lung focus. One of the commonest sequelae in children appears to be spinal caries.

Series of cases followed.-Between 1920 and 1932, 71 cases of pleurisy with effusion in children under 15 years of age were admitted to St. Thomas's Hospital. An attempt was made to trace only those cases in which the presence of the fluid was confirmed by aspiration of which there were 49 . 
The age of onset of the effusion varied from 2 years and 8 months to 15 years of age.

There were 5 cases under 5 years of age, 16 cases between 5 and 10 years of age, and 28 cases between 10 and 15 years of age.

In 21 cases the right side was affected and in 28 cases the left. In no case was the effusion bilateral, though one patient developed an effusion on the other side three weeks after leaving hospital.

Thirty-two cases were in males, and seventeen in females, the average age being exactly the same in both sexes ( 9 years).

In a high proportion of the cases (40.7 per cent.) the effusion was diagnosed in May, June, and July, probably due to infection occurring during a period of lowered resistance following the winter months, and the secondary allergic state developing during the spring and early summer.

There was a family history of some form of tuberculous infection in 26.5 per cent. of the case records.

A history of rheumatism was obtained in four cases (8.2 per cent.), in two of these there was a family history of tuberculosis, in one a brother with no history of rheumatism had had a pleural effusion 6 years previously; the fourth case, however, showed a negative Pirquet reaction at the time of the effusion. In three of the four cases the evidence for tuberculosis was at least as strong as that for rheumatism as a possible cause.

The cases followed were seen from $13 \frac{1}{2}$ years after discharge to 1 year and 1 month after discharge. Of the 49 cases, 32 were traced.

Two patients gave a history of a previous pleural effusion, one 2 months previously and one 3 months previously. The first while in hospital had a swinging temperature up to $103^{\circ}$ for three weeks with no absorption of the fluid; a pneumothorax was induced, the fluid absorbed, and he was perfectly well 5 years and 8 months later. The second had a pyrexia up to $103^{\circ}$ for 3 weeks, then settled for 10 days and had a subsequent rise to $102^{\circ}$ for 8 days; she died of pulmonary tuberculosis $3 \frac{1}{2}$ years later. One had had an attack of erythema nodosum 6 months before his pleural effusion, being Mantoux-positive at that time. Symes ${ }^{10}$ says that one group of cases of erythema nodosum develop an acute attack of tuberculosis, often almost exactly 6 months later, and in these cases, an acute exudative pleurisy is the most frequent manifestation. One child developed the pleural effusion two days after leaving hospital following an attack of measles.

Lymphocytes were in excess in the fluid in all but 3 cases; in one of these the lymphocytes and polymorphonuclear leucocytes were in equal proportions and a culture of micrococcus catarrhalis was obtained. Polymorphonuclear leucocytes were in excess in the other two cases, one showing enlarged hilum glands in the $\mathrm{x}$-ray when seen subsequently. The 
fluid was sterile in all but three cases: in one a culture of micrococcus catarrhalis was obtained and in two staphyloccus albus was found, probably a contamination in each case.

Subsequent history of 32 cases traced.-Of the 49 cases in which an attempt was made to ascertain the subsequent history, 32 were finally traced. Twenty-seven of these were examined in the course of this investigation, two were in other hospitals at the time and reports on their condition were obtained, and two had died in other hospitals which also furnished reports; the last was a doctor's son whose father reported that he had recently been examined and x-rayed. Seventeen were never traced; eight had moved and their whereabouts were unknown to their previous neighbours; seven failed to reply and lived too far away to be visited. One was living too far away to attend, and one was working away from home.

Of the 32 that were traced, two had died, both of pulmonary tuberculosis, and reports from the hospitals concerned stated that one had died 6 years and 7 months after the pleural effusion, both lungs were affected, more particularly the left, the original effusion having been on the right side; the other had died 3 years and 6 months after the effusion, the left lung being extensively involved, the right upper lobe had become involved two months before she died, the original effusion having been on the right side.

One patient examined during the course of the investigation was found to be suffering from tuberculous peritonitis 2 years and 6 months after the effusion. His mother when questioned stated that she had first noticed some enlargement of the abdomen while the child was in a convalescent home some two months after the pleural effusion, but had taken no special note of it. His x-ray showed increased hilar opacity on the right side, the side of the original effusion.

One case happened to be in hospital again at the time of the investigation and so was seen 2 years and 3 months after the effusion, suffering from caries of the dorsal spine; there were no abnormal physical signs in the chest.

One child developed a pleural effusion on the opposite side three weeks after leaving hospital, sterile fluid was aspirated from this effusion also, and the child was perfectly well when seen again 4 years and 7 months after the second attack.

Three showed indefinite signs but no definite evidence of tuberculosis; one of these had copious night sweats and lassitude with indefinite physical signs at the right apex but a negative $x$-ray 1 year and 1 month after discharge; another was very pale and had a troublesome cough with morning expectoration, but no abnormal physical signs in the chest and a negative $\mathrm{x}$-ray when examined 7 years and 7 months after leaving hospital; the third case had a cough with morning expectoration, poor appetite, a slightly diminished percussion note at the right base, a few scattered rhonchi at both bases, 
and an x-ray suggestive of bronchiectasis at the right base when seen 3 years and 1 month after discharge. Two were in other hospitals at the time, one on account of gastro-enteritis which had cleared up completely, this being his first illness since the pleural effusion 12 years and 5 months before, and in him there were no signs or symptoms of any chest trouble or other disease; the other was in hospital following operation for a gangrenous appendix 12 years and 4 months after the pleural effusion, he had contracted syphilis two years before this but had had no other illnesses, his weight had remained steady and he had no abnormal physical signs in the chest and no symptoms beyond those related to his appendicitis.

The only other case not actually examined was a doctor's son whose father reported that he was now perfectly well; he had had no illnesses beyond infectious fevers since his pleural effusion 8 years and 10 months before, he had no abnormal physical signs in the chest and an x-ray of his chest taken one year ago had been quite normal.

The remaining 22 cases that were seen were perfectly well, had had no serious illness since their pleural effusions, and showed no abnormal signs in the chest; four of these showed x-ray evidence of enlarged or calcified hilum glands.

Of the 32 cases, therefore, 25 showed no evidence of subsequent tuberculosis, all being perfectly well except two who were in hospital for some other complaint, three had vague symptoms but not definite evidence of tuberculosis, two had died of pulmonary tuberculosis, one had caries of the dorsal spine, and one had tuberculous peritonitis. Expressed in another way, it is found that 12.5 per cent. subsequently developed tuberculosis, 6.25 per cent. having died of pulmonary tuberculosis, $9 \cdot 4$ per cent. presented doubtful signs and symptoms, and $78 \cdot 1$ per cent. remained perfectly well.

\section{Conclusions.}

1. The immediate prognosis in children having sterile serous pleural effusions is very good.

2. Tuberculosis is a much less frequent sequela of pleural effusions in children than in adults, but occurs sufficiently often to necessitate prolonged treatment and careful supervision of these cases.

3. The dangerous period to the child is the first four years following the effusion and particularly the first year.

4. The commonest sequelae in children appear to be pulmonary tuberculosis and spinal caries.

I am indebted to the physicians of St. Thomas's Hospital for permission to publish these cases and to the Almoner's Department without whose help it would have been impossible to trace many of these patients. 


\section{REFERENCES.}

1. Scheel, O., \& Föien, M., Acta Med. Scand., Stockholm, 1928, LXVIII, 5, (Abstract in Tubercle, London, 1928, IX, 596).

2. Mazzeo, A., La Pediatria, Naples, 1926, XXXIV, 1292.

3. Osler, W., Princ. \& Pract. of Medicine, New York, 1930, 665.

4. Hebert, G. T., Pulmonary Tuberculosis, London, 1927, 128.

5. Norris, G. W., \& Landis, H. R. M., Dis. of Chest and the Princ. of Phys. Diag., Philad., 1929, 646.

6. Graham, S., Glas. Med. Journ., Glasgow, 1925, CIV, 1.

7. Nobel, E., \& Steinebach, R., Ztschr. f. Kinderh., Berlin, 1921, XXXI, 98. (Quoted by Graham, supra.)

8. Neuland, W., Klin. Wchnshr., Berlin, 1922, I, 470. (Quoted by Graham, supra.)

9. Findlay, L., Proc. Roy. Soc. Med., London, 1933, XXV, 410.

10. Symes, J. O., Tubercle, London, 1930, II, 154.

\section{ERRATUM.}

In Dr. K. H. Tallerman's paper on Sensitivity to Cow's Milk Proteins in Acute Gastro-enteritis, Vol. 9, June, 1934, in the table on page 192, column 5, opposite 15. D.O. read (a) \pm instead of (a) + . 